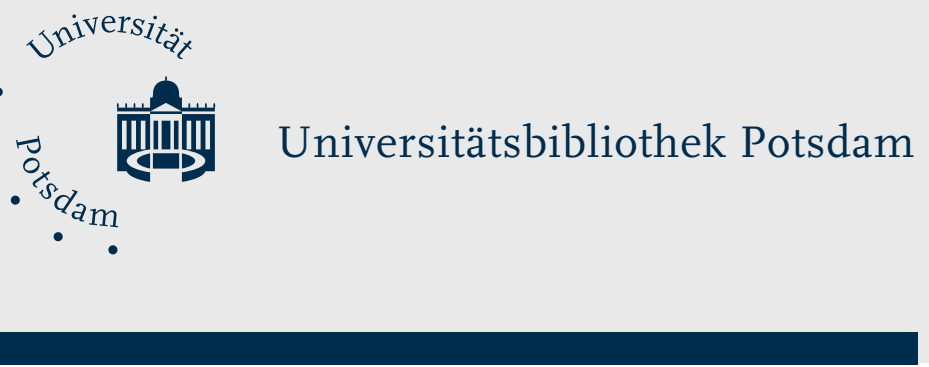

Linda Thomas | Heike Stadler

\title{
Workflow zur Identifizierung von Publikationen für die Zweitveröffentlichung
}

Suggested citation referring to the original publication:

Bibliotheksdienst 50 (2016) 1, S. 62-68

DOI http://dx.doi.org/10.1515/bd-2016-0006 



\section{Linda Thomas und Heike Stadler Workflow zur Identifizierung von Publikationen für die Zweitveröffentlichung}

DOI 10.1515/bd-2016-0006

Zusammenfassung: Verfügbare Allianz- und Nationallizenzen können eine OpenAccess-Komponente beinhalten. Diese räumen den autorisierten Autoren oder Einrichtungen bestimmte Open-Access-Rechte zur Zweitveröffentlichung ein. Vorhandene Lizenzrechte zur Realisierung des Grünen Weges können somit zukünftig verstärkt genutzt werden. Dabei kann die Bibliothek eine aktive Rolle einnehmen. Präsentiert wird ein Workflow, der sich an der Checkliste für Repository-Manager orientiert. Sieben herausgearbeitete Schritte werden genannt und näher erläutert. An Beispielen wurde der Workflow getestet. Die Ergebnisse werden ebenfalls vorgestellt. Die Erweiterung des Workflows zur Identifizierung von Publikationen für die Zweitveröffentlichung lässt sich ggf. auch auf Creative-Commons-Lizenzenanwendenbzw.aufZeitschriften,diekeineOpen-Access-Komponente haben.

Schlüsselwörter: Open Access, Zweitveröffentlichung, Grüner Weg, Elektronische Zeitschriftenbibliothek (EZB), Lizenzen

\section{Workflow for identifying works for secondary publication}

Abstract: Available alliance and national licences may contain an Open Access component. They grant authorised authors or institutions certain Open Access rights for secondary publication. Existing licence rights for realising the Green Road can be used to an increased extent in the future, and the library can play an active role. This article presents a workflow that is oriented towards the check-list for repository managers. It cites seven steps that have been identified and gives more details about them. The workflow has been tested on examples, the results are presented as well. The extension of the workflow for identifying works for secondary publication may perhaps be applicable on Creative Commons licences or magazines that do not have an Open Access component.

Linda Thomas: linda.thomas@uni-potsdam.de Heike Stadler: heike.stadler@uni-potsdam.de 
Keywords: Open Access, secondary publication, Green Road, Electronic Journals Library (Elektronische Zeitschriftenbibliothek, EZB), licences

„Es bleibt zu wünschen, dass die hier verhandelten Rechte und Möglichkeiten umfänglich von den wissenschaftlichen Einrichtungen in Deutschland genutzt und in Anspruch genommen werden“, so Kristine Hillenkötter abschließend in ihrem Aufsatz zur Open-Access-Komponente in den DFG-geförderten AllianzLizenzen. ${ }^{1}$ Genau wie Anja Stöber ${ }^{2}$ erläutert auch Hillenkötter Grundlagen und Herausforderungen zur Anwendung der Open-Access-Komponente und die darin enthaltenen Rechte. Dabei berufen sich beide auf die einschlägige Handreichung zur Thematik. ${ }^{3}$ Praktische Hinweise zur Ausarbeitung eines Workflows befinden sich in allen drei Publikationen, im Detail kann folglich jede Einrichtung selbst einen Geschäftsgang erarbeiten. Der vorliegende Aufsatz knüpft an die genannte Fachliteratur an und präsentiert einen detaillierten, einfach handhabbaren Workflow zur Identifizierung von Publikationen für die Zweitveröffentlichung auf Basis der Open-Access-Komponente in Allianz- und Nationallizenzen. Darüber hinaus wird sich zeigen, dass sich der Workflow problemlos anpassen lässt, wenn z. B. auf Grund von Creative-Commons-Lizenzen (CC-Lizenzen) Zweitveröffentlichungen geplant sind.

Lizenzierte Allianz- und Nationallizenzen können eine Open-Access-Komponente beinhalten, sprich es werden den autorisierten Autoren und/oder Einrichtungen bestimmte Open-Access-Rechte zur Zweitveröffentlichung eingeräumt. Somit können Lizenzrechte für subskriptionspflichtige Publikationen genutzt werden, um den Grünen Weg des Open Access zu fördern. Dabei kann die Bibliothek eine aktive Rolle einnehmen und den Prozess selbst steuern. Im Folgenden werden sieben herausgearbeitete Workflowschritte genannt (vgl. Tab. 1), die sich an der Checkliste für Repository-Manager orientieren. Anschließend werden die einzelnen Phasen näher erläutert und durch Beispiele ergänzt.

1 Hillenkötter, Kristin: Die Open-Access-Komponente in den DFG-geförderten Allianz-Lizenzen. In: Bibliothek Forschung und Praxis, 36 (2012), Dezember, S. 300-304, hier S. 304. DOI 10.1515/ bfp-2012-0039 [Zugriff: 05.10.2015].

2 Stöber, Anja: Open-Access-Rechte in Allianz- und Nationallizenzen. In: Bibliothek Forschung und Praxis, 36 (2012), Dezember, S. 364-368, hier S. 364 ff. DOI 10.1515/bfp-2012-0049 [Zugriff: 05.10.2015].

3 Open-Access-Rechte in Allianz- und Nationallizenzen. Eine Handreichung für Repository-Manager, Bibliothekare und Autoren (2012). DOI 10.2312/allianzoa.004 [Zugriff: 05.10.2015]. 
Tab. 1: Workflow.

\begin{tabular}{ll}
\hline Phase & Workflowschritt \\
\hline Phase 1 & Identifizierung von Allianz- und Nationallizenzen mit Open-Access-Komponente \\
\hline Phase 2 & Ermittlung von Publikationen von Autoren der Einrichtung \\
\hline Phase 3 & $\begin{array}{l}\text { Identifizierung von Zeitschriften aus lizenzierten Allianz- und Nationallizenzen mit } \\
\text { Open-Access-Komponente }\end{array}$ \\
\hline Phase 4 & $\begin{array}{l}\text { Ermittlung von Publikationen von Autoren der Einrichtung innerhalb der Zeitschrif- } \\
\text { ten aus lizenzierten Allianz- und Nationallizenzen mit Open-Access-Komponente }\end{array}$ \\
\hline Phase 5 & Auswertung der Ergebnisse nach Lizenz/Verlag und Open-Access-Rechten \\
\hline Phase 6 & Aufbereitung für die Zweitveröffentlichung \\
\hline Phase 7 & Zweitveröffentlichung \\
\hline
\end{tabular}

$\mathrm{Zu}$ Beginn wird zwangsläufig eruiert, an welchen Allianz- und Nationallizenzen mit Open-Access-Komponente eine Einrichtung teilnimmt (Phase 1). Hilfreich sind neben den Angaben in der Übersicht zur Nutzung der verhandelten Open-Access-Rechte ${ }^{4}$ auch die Lizenzverträge bzw. die Beitrittsinformationen zur Lizenz. Nicht außer Acht lassen sollte man Lizenzen, die einer Moving Wall unterliegen. Nach der Bestandsaufnahme muss die erste relevante Datenbasis ermittelt werden: Publikationen von Autoren der Einrichtung (Phase 2). Mittels der Abfragemöglichkeit „Organization-Enhanced“ in der Datenbank Web of Science kann diese entsprechend aufbereitet werden. Steht eine kostenpflichtige Aufsatzdatenbank nicht zur Verfügung, kann gegebenenfalls auf die Hochschulbibliographie zurückgegriffen werden. Wichtig ist jedoch, dass in dieser ersten Datenbasis eine ISSN vorhanden ist. Abfragen im Web of Science zu unterschiedlichen Zeitpunkten haben ergeben, dass die erste relevante Datenbasis sich ändern kann. Dies hat Auswirkungen auf das Gesamtergebnis.

Für die Realisierung der Phase 3 (Identifizierung von Zeitschriften aus lizenzierten Allianz- und Nationallizenzen mit Open-Access-Komponente) bedient sich die Bibliothek einer einfachen Abrufmöglichkeit im Administrationsbereich der Elektronischen Zeitschriftenbibliothek (EZB)..$^{5}$ In der EZB können Titellisten anhand eines sogenannten Anchor/Ankers abgerufen werden. Ein Anker spiegelt

4 DFG Nationallizenzen. Übersicht zur Nutzung der verhandelten Open-Access-Rechte. https:// www.nationallizenzen.de/open-access [Zugriff: 05.10.2015].

5 Administrationsfunktionen der Elektronischen Zeitschriftenbibliothek, Kapitel 8. http:// rzblx1.uni-regensburg.de/ezeit/admindoc/admin.html [Zugriff: 05.10.2015]. 
i. d. R. alle Zeitschriftentitel einer Lizenz wider. Da Allianz- und Nationallizenzen in der EZB stets zentral verwaltet und gepflegt werden, ist davon auszugehen, dass die Titellisten und Lizenzzeiträume auf einem aktuellen Stand sind. Es wird empfohlen, dass sich Bibliotheken in diesem Workflowschritt alle Anker aller in Frage kommenden Lizenzen aus der EZB ziehen, um am Ende einen Gesamtüberblick über die Zahl der Zweitveröffentlichungen zu erhalten. Parallel kann somit der zeitliche Aufwand für dieses Projekt besser eingeschätzt werden. Die abgerufenen Titellisten ergeben neben der Datenbasis aus Phase 2 folglich die zweite relevante Datenbasis für die anvisierte Auswertung in Phase 4.

Beide Datenmengen werden in Phase 4 (Ermittlung von Publikationen von Autoren der Einrichtung innerhalb der Zeitschriften aus lizenzierten Allianzund Nationallizenzen mit Open-Access-Komponente) mittels MS Excel verarbeitet. Unter Verwendung der ISSN als Suchkriterium im Rahmen des S-Verweises werden die Ankerlisten durchsucht. Auf diese Weise können Publikationen innerhalb lizenzierter Zeitschriften ermittelt werden. Das Suchkriterium muss entsprechend trunkiert werden, damit auch in Zellen mit mehr als einer ISSN (Ankerlisten) ein Ergebnis gefunden werden kann. Damit wird eine Aufsplittung der Zellen mit mehreren ISSN vermieden.

Anschließend erfolgt eine Auswertung der Ergebnisse nach Lizenz und OpenAccess-Rechten (Phase 5). Eine entsprechende tabellarische Aufbereitung verrät der Bibliothek, wie viele Publikationen je Lizenz identifiziert wurden und demzufolge zweitveröffentlicht werden können. Pro Verlag können in dieser Phase die Open-Access-Rechte ausgewertet werden. Unter anderem muss zum Beispiel geklärt werden, welche archivierbare Version zur Veröffentlichung verwendet werden darf. Die Open-Access-Rechte bestimmen die einzelnen Arbeitsschritte in Phase 6 (Aufbereitung für die Zweitveröffentlichung). So kann es durchaus vorkommen, dass der Weg zur archivierbaren Version gegebenenfalls über den Autor führt. Ein Embargo könnte weiterhin die zeitnahe Realisierung des Grünen Weges nach der Erstveröffentlichung verhindern. Nach Überprüfung aller Bedingungen steht einer Zweitveröffentlichung auf einem Repositorium nichts mehr im Wege (Phase 7).

Der erläuterte Workflow wurde bereits bis zur Phase 5 im Dezernat Medienbearbeitung an der Universitätsbibliothek Potsdam entsprechend aufbereitet. Im Folgenden werden drei Beispiele präsentiert, dabei handelt es sich um die Allianz-Lizenz von De Gruyter, Sage und Oxford University Press. ${ }^{6}$ Die erste Datenbasis aus dem Web of Science bilden die Publikationen der Universität Potsdam der Erscheinungsjahre 2011 bis 2014 (zirka 3.890 Ergebnisse).

6 Stand der Abfragen im Web of Science sowie in der EZB für die UB Potsdam: 04.09.2015. 
Für die Auswertung der Schnittmenge auf Basis der ISSN mit der Lizenz des Verlages De Gruyter wurde der EZB-Anker natli_degruyter_llh hinzugezogen, dieser umfasst 161 Zeitschriftentitel. Es wurden insgesamt 18 Artikel identifiziert, die gemäß den vereinbarten Open-Access-Rechten unter Verwendung des Verlags-PDFs umgehend zweitveröffentlicht werden können. Im Rahmen der Allianz-Lizenz des Verlages Sage wurden 30 Publikationen aus 725 Zeitschriften ermittelt, die nach einem Embargo von zwölf Monaten und ebenfalls unter Verwendung der publizierten Fassung für den Grünen Weg freigegeben sind. Den größten Output verzeichnete die Lizenz des Verlages Oxford University Press. Aus 284 Zeitschriftentiteln wurden 142 Publikationen für die Zweitveröffentlichung ermittelt. Leider besagen derzeit die Open-Access-Rechte, dass der Umweg über den Autor eingehalten werden muss, da nur die akzeptierte Version auf einem Repositorium der Wahl veröffentlicht werden darf. Außerdem muss ein Embargo von zwölf bzw. 24 Monaten eingehalten werden. Es bleibt zu hoffen, dass die Open-Access-Rechte bei erneuter Vertragsverhandlung mit Oxford University Press wissenschaftsfreundlicher ausgehandelt werden.

Eine Bestandsaufnahme über alle erworbenen Allianz-Lizenzen hat gezeigt, dass derzeit etwa zehn Prozent der jährlichen Veröffentlichungen auf Basis der Open-Access-Komponente zweitveröffentlicht werden können. Nachfolgende Abbildung verdeutlicht den Workflow noch einmal grafisch.

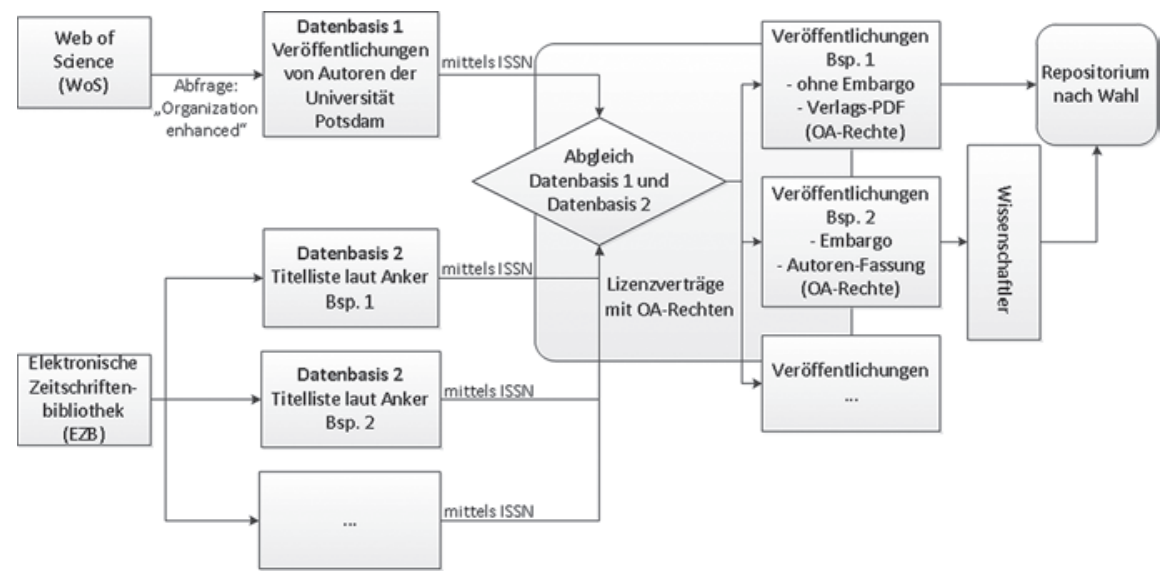

Abb.1: Prozessdarstellung des Workflows.

Wie bereist eingangs erwähnt, lässt sich der ausgearbeitete Workflow auch auf andere Aspekte der Auswertung zwischen publizierten Aufsätzen und Zeitschriften anwenden (z. B. Vorbereitungen auf Lizenzverhandlungen, Zweitveröffent- 
lichungen auf Basis von CC-Lizenzen oder Embargofristen, Publikationsfonds). Hierfür empfiehlt sich ein Gesamtabzug der EZB, welcher unabhängig von Titellisten mittels Anker im Administrationsbereich abgerufen werden kann. Somit können auch Publikationen in nicht lizenzierten Zeitschriften und Open-AccessZeitschriften schnell identifiziert werden.

Mittels dieser angepassten Abfragetechnik konnten an der Universitätsbibliothek Potsdam für die Erscheinungsjahre 2011 bis 2014 exakt 276 Veröffentlichungen ermittelt werden, die auf Basis von CC-Lizenzen auf dem institutionellen Repositorium publiziert werden können (PLOS: 98, BMC: 40, Copernicus: 64, Frontiers: 56, MDPI: 18). Anhand der Zahlen ist zu erkennen, dass der Output steigt.

Tab. 2: Auswertung der Abfrage für die Zweitveröffentlichung auf Basis von CC-Lizenzen.

\begin{tabular}{llcllccl}
\hline & PLOS & BMC & Copernicus & Frontiers & MDPI & gesamt & Anteil \\
\hline Sep. 2015 & 16 & 7 & 16 & 18 & 2 & 59 & $8,55 \%$ \\
\hline 2014 & 33 & 11 & 26 & 20 & 10 & 100 & $9,28 \%$ \\
\hline 2013 & 24 & 13 & 14 & 15 & 2 & 68 & $7,20 \%$ \\
\hline 2012 & 27 & 8 & 12 & 14 & 3 & 64 & $6,41 \%$ \\
\hline 2011 & 14 & 8 & 12 & 7 & 3 & 44 & $5,10 \%$ \\
\hline
\end{tabular}

Es empfiehlt sich, die Realisierung des genannten Workflows zunächst als Projekt in der Einrichtung anzulegen und dann jährlich in eine Routinebearbeitung zu wechseln. So kann gewährleistet werden, dass die Lizenzmöglichkeiten (Open-Access-Komponente, Embargofristen, CC-Lizenzen) im vollen Umfang ausgeschöpft werden. 


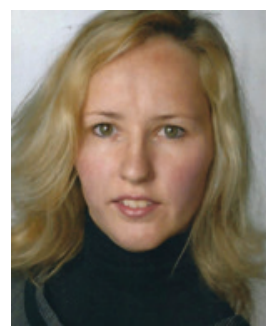

Linda Thomas M.A., M.A. (LIS)

Universitätsbibliothek Potsdam

Dezernat Medienbearbeitung

Am Neuen Palais 10

14469 Potsdam

Tel.: 0331-977-2517

E-Mail: linda.thomas@uni-potsdam.de

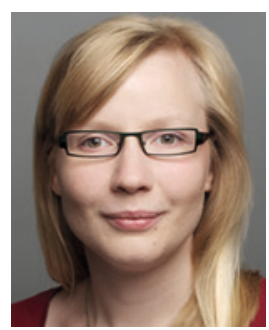

\section{Dipl.-Bibl. Heike Stadler}

Universitätsbibliothek Potsdam

Dezernat Medienbearbeitung

Am Neuen Palais 10

14469 Potsdam

E-Mail: heike.stadler@uni-potsdam.de 